\title{
POSSIBILITIES OF USING OUTPATIENT PHYSIOTHERAPY IN THE PROCESS OF REHABILITATION OF PEOPLE WITH DEGENERATIVE DISC DISEASE IN THE LUMBOSACRAL SPINE
}

\author{
Bartosz Bolach ${ }^{A, E, C}$ \\ Faculty of Physical Education and Sport, Department of Paralympic Sports, Academy of Physical Education in Wroclaw, Poland \\ ORCID: 0000-0002-3433-4434
}

Jagoda Walowska ${ }^{\mathrm{B}, \mathrm{D}}$

RB Dr Jagoda Walowska, Wroclaw, Poland

ORCID: 0000-0002-0130-0211 | E-mail: jagodawalowska@wp.pl

\section{Paulina Chabraszewska ${ }^{A, B, C}$}

Institute of Physiotherapy, University of Physiotherapy in Wroclaw, Poland

\author{
Karina Ryterska ${ }^{\mathrm{D}}$ \\ Pomeranian Medical University in Szczecin, Poland \\ ORCID: 0000-0003-2791-3312
}

\section{Eugeniusz Bolach ${ }^{\mathrm{D}} \mathrm{E}$}

Faculty of Physical Education and Sport, Department of Paralympic Sports, Academy of Physical Education in Wroclaw , Poland ORCID: 0000-0003-1857-0522

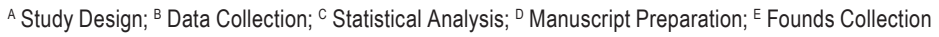

\begin{abstract}
Ahstract Degenerative disc disease (DDD) in the lumbosacral spine is one of the most common causes of pain and the significant associated limitations in physical activity and daily functioning, with the vast majority of patients requiring long-term physiotherapy. Hence, the significance of proper diagnostics, locating the cause of the ailment, implementation of appropriate therapy and prevention.

The aim of the study was to investigate the efficacy of outpatient physiotherapy on reducing pain and improving the function of the lumbosacral spine. The research group comprised 95 people (50 women and 45 men) with an average age of 53 years, all patients with DDD in the lumbosacral spine. They underwent 3 physical treatments: magnetotherapy, laser therapy, and systemic cryotherapy, as well as gymnastic exercises, aimed at improving physical fitness, and strengthening the muscular corset. The research methods included the Schober test, the Thomayer test (finger-ground test), the Visual Analogue Scale scale, Laitinen's pain questionnaire, and calculation of BMI.

Physiotherapeutic treatments significantly reduced the patients' pain symptoms, significantly increased the range of motion in the lumbosacral spine and improved physical fitness. Better results of the therapy were observed in patients with lower BMI.
\end{abstract}

Key Words intervertebral disc, fibrous annulus, nucleus pulposus, spine 


\section{Introduction}

Degenerative disc disease (DDD) in the lumbosacral spine is one of the most common causes of pain and significant associated limitations in physical activity and everyday functioning. It is estimated that this condition affects $12-35 \%$ of the human population. The lumbosacral spine is particularly vulnerable to injuries and can overload as a result of everyday activities. The intervertebral disc can be damaged, resulting in displacement of the pulmonary nucleus, along with irritation of other anatomical structures located within the lesion, such as joint capsule, interspinal and longitudinal ligaments, paraspinal muscles, nerve root sheaths, dura mater, vertebral body, and connective tissue in the area of the lesion within nerves and blood vessels. However, only a small percentage of patients require surgery. The vast majority qualify for conservative treatment aimed at reducing both the pain and the negative consequences to physical fitness (Colombini, Lombardi, Corsi, Banfi, 2008; Roughley, 2004; Frost, Camarero-Espinosa, Foster, 2019). When long-term pain contributes to the occurrence of chronic discomfort in the sacral spine and the entire back, treatment and therapy require more time and increased economic outlays (Hodgkinson, Shen, Diwan, Hoyland, Richardson, 2019).

Many factors can affect the integrity of the intervertebral disc. These include mechanical and genetic factors, past injuries, and diet. Progressive degenerative processes include structural damage to the intervertebral disc and changes in cell number and composition. A major factor in the degeneration of the intervertebral disc is the loss of proteoglycans. Degenerative changes in the disc are also associated with damage to adjacent structures which lead to functional changes, such as increased susceptibility to injuries and increased clinical symptoms (Kos, Gradisnik, Velnar, 2019).

There is a correlation between the location of the occurrence of back pain and age, sex and changes observed in the MRI scan. Male intervertebral discs degenerate more than women in the second, third, fourth, and even seventh decades of life. The most common degenerative changes concern the vertebrae L4-S1. Additional predisposing factors include a sedentary lifestyle, smoking and obesity (Cheung at al., 2009; Kanayama, Togawa, Takahashi, Terai, Hashimoto, 2009; Miller, Schmatz, Schultz, 1988; Like at al., 2005). Due to the nature and extent of the changes, as well as the prolonged pain which significantly limits the ability to function freely, these patients sometimes require long-term leave from work and rehabilitation, one of the main assumptions of which is to minimize pain and improve functioning in everyday life Peolsson at al., 2014; Newell et al., 2017). The vast majority of patients require long-term physical therapy. That is why proper diagnostics, locating the cause of the ailment, and implementing appropriate therapy and prevention are so important (Urban, Roberts, 2003).

\section{Aim of the study}

The aim of the study was to investigate the efficacy of outpatient physiotherapy on reducing pain and improving spine function in patients with DDD in the lumbosacral spine.

\section{Research questions}

1. To what extent did the mobility of the lumbosacral spine improve after outpatient rehabilitation?

2. Is the feeling of pain after outpatient rehabilitation different between sexes? 


\section{Material}

The research was conducted at the "CREATOR" Non-Public Healthcare Center in Wrocław. 50 women and 45 men, aged 46 to 60 years, were examined. The age distribution of the men and women was very similar. The mean age of the women was 53.7 years with a standard deviation of 4.2 years, while the mean age of the men was 53.5 years with a standard deviation of 4.7 years. The average weight of the women was $68.8 \mathrm{~kg}$, and the men $91.4 \mathrm{~kg}$. Importantly, the mean BMI values of the women and men were within the range of overweight. The difference in the BMI distributions of women and men was statistically significant. Women were characterized by a higher level of education than the men. They also performed office work more often.

\section{Methods}

The orthopedic doctor selected 95 people diagnosed with pain due to DDD. Each patient before and after the rehabilitation cycle was examined using the Schober test and Thomayer test (finger-ground test), and the level of pain perception was assessed. Results were processed using the Visual Analogue Scale (VAS) and the Laitinen's Pain Questionnaire.

The scope of the outpatient physiotherapy used included 20-minute magnetotherapy treatment at a frequency from $0 \mathrm{~Hz}$ to $50 \mathrm{~Hz}$ and a field of $6 \mathrm{mT}$ to $10 \mathrm{mT}$, trigger point laser therapy $\left(1 \mathrm{~cm}^{2}\right)$ with a dose of $6-9 \mathrm{~J} / \mathrm{cm}^{2}$, systemic cryotherapy in a cryochamber (from $-60^{\circ} \mathrm{C}$ to $-130^{\circ} \mathrm{C}$ ). The clinical therapy procedures were followed by classes in systemic gymnastics aimed at reducing back pain and strengthening the postural muscles responsible for the correct body posture and stabilization of the thoracic-lumbar spine. The gymnastics began with a 10-minute warm-up on a cyclo-ergometer, then the patients participated in a 20-minute group gymnastic exercise comprising 14 flexion exercises.

\section{The set of exercises}

1. Lying on the back, upper limbs along the body, lower legs straight. Exercise: alternate bending of the lower limbs by grasping the knee joint and pulling it towards the chest while lifting the head.

2. Item as above. Exercise for bilateral bending of the lower limbs by grasping both knee joints and pulling them to the chest while raising the head at the same time.

3. In the supine position, upper limbs downwards obliquely, lower limbs bent at an angle of about 60 degrees. Exercise for alternating sideways twisting of both lower limbs.

4. In the supine position, the upper limbs along the body, the lower limbs bent at an angle of about 60 degrees. Exercise to lift the torso up and down.

5. Lying on the back, upper limbs along the body, lower legs straight. Exercise for bending the upper and lower limbs with a simultaneous deep inhalation through the nose and extension of the limbs with a simultaneous longer exhalation.

6. In the front lying position, chin rested on a mattress, upper limbs along the body, lower legs straightened. Exercise of alternating lower limb lifting.

7. In the front lying position, chin rested on the mattress, upper limbs up, lower legs straight. Exercise of alternating lifting of the upper left and lower limb right and upper right and lower left limb. 
8. In the front lying position, chin resting on the mattress, upper limbs up, lower legs straightened. Exercise for simultaneous lifting of upper and lower limbs. Kneeling, propped up. Exercise of alternating lower limb lifting.

9. Kneeling, propped up. Exercise of alternating lifting of the upper left and lower limb right and upper right and lower left limb.

10. Slow kneeling, the arms along the body. Exercise for lifting the upper limbs while inhaling deeply through the nose and lowering them downward while exhaling for a longer time.

11. Free kneeling. Left torso twist exercise, while grasping the left hand for the left heel and the right hand for the right heel.

12. One-legged slow kneeling, the other lower limb straightened at the knee joint. Exercise of lifting both upper limbs and bending the torso to the straightened lower limb and returning to the starting position, and then changing the position of the limbs.

13. In a standing position, lifting the upper limbs, inhaling through the nose and exhaling through the mouth. Each exercise was performed for 10 repetitions. Patients tried to perform 3 series of exercises, of which breathing exercises were performed up to 6 repetitions.

\section{Statistical analysis}

In the statistical description of the research material, mean values, standard deviations and the range of variability of the feature were used. The statistical significance of changes in the examined features from the performed physiotherapeutic procedures was assessed using the Student's t-test for dependent samples. While the null hypothesis about the lack of influence of physiotherapy on the distribution of the analyzed trait being true, the Student t-distribution with $\mathrm{N}-1$ degrees of freedom was true, on the basis of which the significance level $p$ was determined. Sexual dimorphism of the analyzed traits was tested using the Student's t-test for independent samples. If the null hypothesis about the lack of sex-related differentiation of the distributions of the analyzed feature was true, we tested the t-Student's distribution with $\mathrm{N} 1+\mathrm{N} 2-2$ degrees of freedom. The relationship between the distribution of BMl values and gender was assessed using the chi square $\left(\mathrm{X}^{2}\right)$ test of independence. Taking into account the division of BMI values into three categories and the validity of the null hypothesis about the lack of a relationship between sex and BMI distribution, the $x^{2}$ test function had a Pearson chi-square distribution with two degrees of freedom. When verifying the null hypotheses, a critical significance level $\alpha=0.05$ was used. The calculations were performed using STATISTICA v.10 package by StatSoft (Shapiro, Wilk, 1965; Siegel, 1956).

\section{Results}

In the Schober test, all patients obtained better results after using outpatient physiotherapy. Before the start of the treatment cycle, the total percentage of people, regardless of gender, with the correct result $(i . e .5-7 \mathrm{~cm}$ ) in the entire study group was $20.5 \%$ (women $28 \%$ and men $13 \%$ ), which almost doubled after physiotherapy at $38.5 \%$ ( $50 \%$ for women, $27 \%$ for men). The mean Schober test results in the group of men were worse than in the group of women, both in the initial study and after physiotherapy. The difference in mean results in both sex groups was at the borderline of statistical significance in the initial study $(p=0.06 \approx 0.05)$, and after the applied physiotherapy it was statistically significant.

When analyzing the Thomayer test, it was noticed that in the initial study, before the start of the cycle of physiotherapeutic treatments, men achieved significantly worse results than women. The difference in mean 
results could not be considered statistically significant at the adopted level of significance $(p=0.073>0.050)$, but $p$ was close to the critical value of $a=0.05$. The result of the Thomayer test correlated significantly with the BMI value. The rank coefficient of Spearman's BMI and the result of this test in the initial study was $\rho=-0.42$. The correlation was negative, which meant that higher BMI values were not conducive to achieving good results in this test. Therefore, the worse results of men were a consequence of higher BMI values compared to those achieved by the women. Also after physiotherapy, the mean score of men was still lower than the mean score of women, but the difference in mean values in both groups lessened. The improvement of the Thomayer test result after outpatient physiotherapy was strongly negatively correlated with the test result obtained before the start of the treatment cycle. The Spearman's rank correlation coefficient was in this case $\rho=-0.76$. This explains why the improvement was greater in the group of men. The negative correlation indicated that greater improvement should be expected in people who had worse results in the Thomayer test before the therapy, and such people were mainly men.

Analyzing the pain scale, it was found that the applied forms and types of therapeutic rehabilitation contributed to a reduction in the subjective level of experiencing pain. The mean value of pain intensity was significantly higher in the group of men, both in the initial and final study. The applied outpatient physiotherapy decreased the mean value of pain by more than 4 points on the VAS scale. This change was highly statistically significant. The mean pain reduction was virtually independent of gender. In the group of women, the mean change in the intensity of perceived pain was 4.12 on the VAS scale, and in the group of men it was 4.22 .

Tahle 1. Comparison of the results achieved in tests and scales in the group of women and men before and after outpatient physiotherapy

\begin{tabular}{|c|c|c|c|c|c|c|c|}
\hline \multirow{2}{*}{ Test } & \multirow{2}{*}{ Sex } & \multirow{2}{*}{$\bar{x}$} & \multirow{2}{*}{ Dev. Std. } & \multirow{2}{*}{ Minimum } & \multirow{2}{*}{ Maximum } & \multicolumn{2}{|c|}{ Student test } \\
\hline & & & & & & $t$ & $p$ \\
\hline \multicolumn{8}{|c|}{ Schober test [cm] } \\
\hline \multirow{2}{*}{ Before physiotherapy } & women & 3.43 & 1.54 & 1.0 & 7.0 & \multirow{2}{*}{1.90} & \multirow{2}{*}{0.061} \\
\hline & men & 2.89 & 1.21 & 1.0 & 5.0 & & \\
\hline \multirow{2}{*}{ After physiotherapy } & women & 4.45 & 1.29 & 2.0 & 7.0 & \multirow{2}{*}{2.53} & \multirow{2}{*}{0.013} \\
\hline & men & 3.79 & 1.25 & 1.0 & 6.0 & & \\
\hline \multicolumn{8}{|c|}{ Toe-to-floor test [cm] } \\
\hline \multirow{2}{*}{ Before physiotherapy } & women & -9.9 & 13.0 & -60 & 15 & \multirow{2}{*}{1.82} & \multirow{2}{*}{0.073} \\
\hline & men & -14.4 & 10.8 & -46 & 3 & & \\
\hline \multirow{2}{*}{ After physiotherapy } & women & -4.1 & 8.7 & -35 & 15 & \multirow{2}{*}{1.55} & \multirow{2}{*}{0.126} \\
\hline & men & -7.0 & 9.5 & -39 & 8 & & \\
\hline \multicolumn{8}{|c|}{ Pain on the VAS scale [pts] } \\
\hline \multirow{2}{*}{ Before physiotherapy } & women & 6.80 & 1.60 & 3.0 & 10.0 & \multirow{2}{*}{2.87} & \multirow{2}{*}{0.005} \\
\hline & men & 7.78 & 1.72 & 4.0 & 10.0 & & \\
\hline \multirow{2}{*}{ After physiotherapy } & women & 2.68 & 1.73 & 0.0 & 7.0 & \multirow{2}{*}{2.62} & \multirow{2}{*}{0.010} \\
\hline & men & 3.56 & 1.50 & 0.0 & 7.0 & & \\
\hline \multicolumn{8}{|c|}{ Laitinen pain questionnaire [pts] } \\
\hline \multirow{2}{*}{ Before physiotherapy } & women & 6.7 & 1.9 & 4.0 & 12.0 & \multirow{2}{*}{3.49} & \multirow{2}{*}{0.001} \\
\hline & men & 8.2 & 2.1 & 4.0 & 13.0 & & \\
\hline \multirow{2}{*}{ After physiotherapy } & women & 2.5 & 1.8 & 0.0 & 8.0 & \multirow{2}{*}{3.25} & \\
\hline & men & 3.7 & 1.9 & 1.0 & 8.0 & & 0.002 \\
\hline
\end{tabular}


The performed physiotherapeutic procedures reduced the level of perceived pain, as assessed with the Laitinen pain questionnaire. The observed changes were highly statistically significant. The mean reduction in pain experienced was only slightly greater in the male group. The effects of the performed physiotherapy in terms of the reduction of pain intensity on the VAS scale as well as the reduction of pain perception on the Laitinen scale were similar. The rank correlation coefficient for both these features was $\rho=0.60$ and was statistically highly significant $(p<0.0001)$. The Laitinen questionnaire assessed not only the intensity and frequency of pain, but also the physical limitations caused by the pain and the number of pharmaceuticals used. Table 1 compares the results achieved in individual tests and scales, in both the groups of women and men, before starting outpatient physiotherapy and after completing the 10-day cycle of physiotherapy. Table 2 shows the average changes in the results achieved in the individual groups of the studied women and men.

Table 2. The average change in test results and the feeling of pain before and after outpatient physiotherapy

\begin{tabular}{|c|c|c|c|c|c|c|c|c|}
\hline \multirow{2}{*}{ Test/scale } & \multirow{2}{*}{ Sex } & \multicolumn{2}{|c|}{ Before physiotherapy } & \multicolumn{2}{|c|}{ After physiotherapy } & \multirow{2}{*}{$\begin{array}{l}\text { Average } \\
\text { change }\end{array}$} & \multicolumn{2}{|c|}{ Student test } \\
\hline & & $\bar{x}$ & SD & $\overline{\mathrm{x}}$ & SD & & $t$ & $P$ \\
\hline \multirow{3}{*}{ Schober test [cm] } & women & 3.43 & 1.54 & 4.45 & 1.29 & 1.02 & 13.75 & $<0.0001$ \\
\hline & men & 2.89 & 1.21 & 3.79 & 1.25 & 0.90 & 17.40 & $<0.0001$ \\
\hline & both sexes & 3.17 & 1.41 & 4.14 & 1.31 & 0.96 & 20.81 & $<0.0001$ \\
\hline \multirow{3}{*}{ Thomayer test [cm] } & women & -9.90 & 13.00 & -4.10 & 8.70 & 5.80 & 7.62 & $<0.0001$ \\
\hline & men & -14.40 & 10.80 & -7.00 & 9.50 & 7.40 & 16.49 & $<0.0001$ \\
\hline & both sexes & -12.00 & 12.20 & -5.40 & 9.10 & 6.60 & 14.30 & $<0.0001$ \\
\hline \multirow{3}{*}{ Pain on the VAS scale [pts] } & women & 6.80 & 1.60 & 2.68 & 1.73 & 4.12 & 24.86 & $<0.0001$ \\
\hline & men & 7.78 & 1.72 & 3.56 & 1.50 & 4.22 & 21.30 & $<0.0001$ \\
\hline & both sexes & 7.26 & 1.72 & 3.09 & 1.68 & 4.17 & 32.68 & $<0.0001$ \\
\hline \multirow{3}{*}{ Laitinen pain questionnaire [pts] } & women & 6.72 & 1.88 & 2.50 & 1.82 & 4.22 & 28.27 & $<0.0001$ \\
\hline & men & 8.16 & 2.12 & 3.73 & 1.88 & 4.42 & 21.87 & $<0.0001$ \\
\hline & both sexes & 7.40 & 2.12 & 3.08 & 1.94 & 4.32 & 34.90 & $<0.0001$ \\
\hline
\end{tabular}

\section{Discussion}

The aging of the populations in the West and elsewhere has been associated with a proportional increase in the incidence of conditions of the intervertebral disc. These conditions are the main cause of back pain limiting to a large extent everyday functioning. That is why it is so important to conduct an accurate diagnosis, which is of key importance for further treatment.

A thorough understanding of the pathophysiology and clinical symptoms helps in recognizing and locating the cause of these conditions. There is extensive literature on the treatment of intervertebral disc conditions. Most often, we treat this ailment by focusing on relieving pain through the use of conservative treatment. Other methods are surgical treatment and the use of molecular therapy, injections in lesions, or the use of stem cell treatment (Wu, Kim, Jang, 2020; Barakat, Elwell, Lam, 2019). In a review of 41 research studies on a total of 6,858 patients, Kamper et al. (2015) showed that sixteen of them provided moderate-quality evidence that multidisciplinary rehabilitation reduced pain (standardized mean difference $0.21-95 \%$ confidence interval 0.04 to 0.37 ; equivalent to 0.5 point on a 10 point pain scale) and disability as a consequence of these conditions $(0.23,0.06$ to 0.40 ; equivalent to 1.5 points on a Roland-Morris 24-point index), compared to usual care not involving physiotherapy. Eight studies 
provided moderate-quality evidence that multidisciplinary rehabilitation increases job prospects one year after the intervention (odds ratio 1.87-95\% confidence interval 1.39-2.53). Two studies comparing multidisciplinary rehabilitation with surgery found a slight difference in outcomes and an increased risk of side effects associated with surgery. This means that properly planned and carried out rehabilitation is extremely important in the rehabilitation process in people with degenerative disc disease (DDD).

Treatments should be performed systematically and appropriately selected according to the ailment and its cause (Kamper at al., 2015). As early as in the 1980s, Zylbergold and Piper (1981) showed in their monthly studies that both the use of exercises aimed at ailments related to the lumbar spine, as well as manual therapy procedures, systematic home visits and patients exercising at home, are effective in the treatment of DDD. Adamczyk, Kiebzak, Wilk-Frańczuk, Śliwiński (2009) compared the results of two different physiotherapeutic approaches in the treatment of back pain in 60 patients. In the study group, an individual treatment program was applied, including post-isometric muscle relaxation (PIR), Kibler Fold spine mobilization, Kinesiology Taping and relaxation exercises. The control group, on the other hand, underwent electrotherapy and performed a set of general development exercises. Pain intensity and difficulty in carrying out daily activities were assessed at the beginning and end of treatment. The measurements included spine mobility, static balance, muscle and ligament tenderness and tension, and the identification of areas with pain ailments. As a result of the intervention, a significant reduction in back pain was found ( $90 \%$ of patients in the study group), and in ( $80 \%$ of patients) balance, muscle and ligament tension, and spine mobility improved. In the control group, radiating pain decreased by $(25 \%)$, and the remaining parameters did not change significantly. The results were statistically significant at $p<0.05$. The authors of this publication, and also Fronczyk and Kuliński (2017), came to the conclusion that physiotherapy is the basis for rehabilitation of patients with back pain syndromes. Fronczyk and Kuliński (2017) analyzed the possibility of using physiotherapy in patients with lumbosacral DDD. The study included a group of 54 patients aged 20-60 years. The research tool was a questionnaire prepared by the authors. The questionnaire included 17 closed and 5 open questions. The results were assessed using two subjective pain intensity scales: the VAS scale and the Laitinen pain index. Moreover, the Schober symptom was used to assess lumbosacral mobility. The conducted research showed that rehabilitation performed in patients reduced pain and improved spine mobility, they took painkillers less frequently and could undertake more frequent and more intense physical activity. Krawczyk-Suszek, Szlichta-Kołeczek, Bednarski (2018) emphasized that the concept of DDD in the lumbosacral spine encompasses all pathological changes within the intervertebral disc which cause characteristic clinical symptoms, mainly pain in the lower part of the spine, often radiating to the lower limbs, and also tingling, numbness and limited mobility of the torso. Their study included 140 people diagnosed with DDD. The research method was a proprietary questionnaire supplemented with the VAS scale. The tests were performed before and after rehabilitation. The data was analyzed statistically. Among the treatments used in patients, exercises aimed at strengthening the lumbar spine and abdominal muscles dominated. In addition, therapeutic massage, TENS currents and manual therapy were used. The mean pain intensity before rehabilitation was 7.21 points \pm 1.54 points, while after rehabilitation $=3.44$ points \pm 1.97 points $(p<0.001)$. Outpatient physiotherapy reduced the pain and contributed significantly to the improvement of the patients' quality of life.

In a study in Hungary, one of the non-invasive methods used to reduce pain in the lumbar spine was traction under controlled conditions. The therapy focused on an attempt to position the spine in the correct position while maintaining the curvature of the spine, which was to affect the reposition of the incorrectly positioned intervertebral disc. It should be emphasized that it was not a universal form of therapy that can be performed in every patient. 
Physical exercise and physical activity undertaken by patients with DDD is extremely important, but as França, Burke, Hanada, Marques (2010) emphasize, better results are achieved by people who perform targeted exercises to strengthen the deep muscles and stabilize the lumbar spine. Performing exercise of a systemic nature has a global impact, contributing to a reduction in pain, moreover, the therapeutic effect is shorter. This study and the research by Plaskiewicz, Kałużny, Kochański (2015) reached similar conclusions, and extended the rehabilitation program additionally with physical therapy. They found that the use of physiotherapeutic procedures, including cryotherapy, electrotherapy, laser therapy, magnetotherapy, ultrasound and heat therapy are particularly effective in the treatment of DDD.

Regardless of the cause and course of DDD, particular attention should be paid to prophylaxis and lifestyle changes related primarily to a significant reduction in the sitting position during the day (Kwon at al., 2018). The positioning of the lumbosacral spine is a key factor for the proper functioning of the spine. Changes in the position of the spine lead to disturbances in the biomechanics of the body and gait. This affects the quality of life, body balance and increases the risk of falls in the elderly (Kocyigit, Berk, 2018). Back pain patients show a variety of kinetic changes when walking on flat ground and when climbing stairs. Both adaptation strategies put additional strain on the spine and joints and additionally increase the risk of developing disc conditions. Kuai et al. (2017) in the gait analysis laboratory compared the gaits of people with pain and in healthy adults. They found that patients with DDD showed significant compensation when performing movements and higher activity of the trunk muscles both when walking on flat ground and climbing stairs. There was a decrease in the anterior-posterior shear forces acting on the discs in the pathological area and an increase in compressive forces on all lumbar vertebrae when walking on flat ground. When climbing stairs, shear forces dominated, which is why, among others, Azadinia, EbrahimiTakamjani, Kamyab, Parnianpour, Asgari (2017) conducted long-term research on the type of physiotherapeutic intervention and its impact on body posture and the way of moving. Pain located in the lumbar spine is one of the most frequently diagnosed condition entities and one of the most frequently reported ailments and the cause of the inability to work. The causes of non-specific pain syndromes are myofascial overloads, ligament injuries, and psychogenic factors. Most often, the cause of the ailment is difficult to pinpoint. In turn, specific pain syndromes are most often caused by hernia of the nucleus pulposus, spondylolisthesis, stenosis of the spinal canal, degenerative changes in the inter-process joints, vertebral fractures, spine tumors or inflammatory diseases. Pain may be perceived as dull, diffuse, with a burning or stinging sensation. They can also cause radiating pain. DDD and related pain syndromes generate huge financial costs, including those for the state budget. They require long-term diagnostics, treatment and rehabilitation, which is associated with the payment of unemployment benefits and pensions. Therefore, the cooperation of the entire therapeutic team is recommended in order to diagnose as soon as possible and obtain positive treatment results (Manek, McGregor, 2005; Rubin, 2007).

\section{Conclusions}

1. In the Schober phenomenon, the subjects showed an improvement in the mobility of the lumbosacral spine in both groups. Women achieved statistically better results.

2. In the Thomayer test, there was a negative correlation with BMI values, which resulted in better results for men.

3. The 10-day therapy significantly increased the range of mobility of the lumbosacral spine in all the patients studied. 
4. Physiotherapeutic procedures significantly reduced the patients' pain symptoms by an average of more than 4 points on the VAS scale. The mean pain reduction was not biased by gender.

5. According to the Laitinen questionnaire, the values of perceived pain were higher in men both in the initial examination and after the application of physiotherapy.

6. Physical limitations caused by pain and the number of painkillers used have reduced significantly after physiotherapy.

7. Better results of the therapy were observed in patients with lower BMI values.

8. Men showed higher values of BMI. In a group where there was not a single subject with a normal body mass BMI, as opposed to 14 subjects.

\section{References}

Adamczyk, A., Kiebzak, W., Wilk-Frańczuk, M., Śliwiński, Z. (2009). Effectiveness of holistic physiotherapy for low back pain. Ortop Traumatol Rehabil., 11 (6), 562-576.

Azadinia, F., Ebrahimi-Takamjani, I., Kamyab, M., Parnianpour, M., Asgari, M. (2017). A RCT comparing lumbosacral orthosis to routine physical therapy on postural stability in patients with chronic low back pain. Med J Islam Repub Iran, 31, 26.

Barakat, A., Elwell, V.A., Lam, K.S. (2019). Stem cell therapy in discogenic back pain. J Spine Surg, 5 (4), 561-583.

Cheung, K., Karppinen, J., Chan, D., Ho, D., Song, Y-Q., Sham, P., Cheah, K., Leong, J., Luk, K. (2009). Prevalence and pattern of lumbar magnetic resonance imaging changes in a population study of one thousand forty-three individuals. Spine, 34 (9), 934-940.

Colombini, A., Lombardi, G., Corsi, M.M., Banfi, G. (2008). Pathophysiology of the human intervertebral disc. Int J Biochem Cell Bio, $40(5), 837-842$.

França, F.R., Burke, T.N., Hanada, E.S., Marques, A.P. (2010). Segmental stabilization and muscular strengthening in chronic low back pain: a comparative study. Clinics, 65 (10), 1013-1017.

Fronczyk, K., Kuliński, W. (2017). Lumbosacral discopathy: analysis of physical therapy. Medical studies, 33 (1), 9-16.

Frost, B., Camarero-Espinosa, S., Foster, E.J. (2019). Materials for the Spine: Anatomy, Problems, and Solutions. Materials, 12 (2), 253.

Hodgkinson, T., Shen, B., Diwan, A., Hoyland, J.A., Richardson, S.M. (2019). Therapeutic potential of growth differentiation factors in the treatment of degenerative disc diseases. JOR Spine, 2 (1), 1045.

Kamper, S.J., Apeldoorn, A.T., Chiarotto, A., Smeets, R., Ostelo, R., Guzman, J., van Tulder, M.W. (2015). Multidisciplinary biopsychosocial rehabilitation for chronic low back pain: Cochrane systematic review and meta-analysis. BMJ, 350, 444.

Kanayama, M., Togawa, D., Takahashi, C., Terai, T., Hashimoto, T. (2009). Cross-sectional magnetic resonance imaging study of lumbar disc degeneration in 200 healthy individuals. J Neurosurg Spine, 11 (4), 501-507.

Kocyigit, B.F., Berk, E. (2018). Comparison of Lumbosacral Alignment in Geriatric and Non-Geriatric patients suffering low back pain. Pak J Med Sci., 34 (2), 282-287.

Kos, N., Gradisnik, L., Velnar, T.A. (2019). Brief Review of the Degenerative Intervertebral Disc Disease. Review/Med Arc., 73 (6), 421-424.

Krawczyk-Suszek, M., Szlichta-Kołeczek, K., Bednarski, J. (2018). Efficacy of rehabilitation in patients with segmental discopathy lumbar-sacral spine. World Scientific News, 111, 40-50.

Kuai, S., Liao, Z., Zhou, D.G.W., Guan, X., Ji, R., Zhang, R., Guo, D., Liu, W. (2017). The Effect of Lumbar Disc Herniation on Musculoskeletal Loadings in the Spinal Region During Level Walking and Stair Climbing. Med Sci Monit., 23, 3869-3877.

Kwon, Y., Kim, Ji.W., Heo, J.H., Jeon, H.M., Choi, E.B., Eoma, G.M. (2018). The effect of sitting posture on the loads at cervico-thoracic and lumbosacral joints. Technol Health Care, 26 (1), 409-418.

Like, M., Solovieva, S., Lamminen, A., Luoma, K., Leino-Arjas, P., Luukkonen, R., Riihimäki, H. (2005). Disc degeneration of the lumbar spine in relation to overweight. Int J Obes., 29 (8), 903-908.

Manek, N.J., MacGregor, A.J. (2005). Epidemiology of back disorders: prevalence, risk factors, and prognosis. Curr Opin Rheumatol, 17 (2), 134-140. 
Miller, J., Schmatz, C., Schultz, A. (1988). Lumbar disc degeneration: correlation with age, sex, and spine level in 600 autopsy specimens. Spine., $13(2), 173-178$.

Newell, N., Little, J.P., Christou, A., Adams, M.A., Adam, C.J., Masouros, S.D. (2017). Biomechanics of the human intervertebral disc: A review of testing techniques and results. Journal of the Mechanical Behavior of Biomedical Materials, 69, 420-434.

Peolsson, A., Öberg, B., Wibault, J., Dedering, A., Zsigmond, P., Bernfort, L., Kammerlind, A.S., Persson, L.C.G., Löfgren, H. (2014). Outcome of physiotherapy after surgery for cervical disc disease: a prospective randomised multi-centre trial. BMC Musculoskelet Disord, 15,34 .

Plaskiewicz, A., Kałużny, K., Kochański, B. (2015). The use of physical therapy in the treatment of pain lumbar spine. Journal of Education, Health and Sport, 5 (5), 11-20.

Roughley, P. (2004). Biology of intervertebral disc aging and degeneration: involvement of the extracellular matrix. Spine, 29 (23), 2691-2699.

Rubin, D.I. (2007). Epidemiology and risk factors for spine pain. Neurol Clin., 25 (2), 353-371.

Shapiro, S, Wilk, M. (1965). An analysis of variance test for normality (complete samples). Biometrika, 52, 591-611.

Siegel, S. (1956). Nonparametric statistics for the behavioral sciences. New York: McGraw-Hill Book Company.

Urban, J.P.G., Roberts, S. (2003). Degeneration of the intervertebral disc. Arthritis Res Ther., 5 (3), 120-130.

Wu, P.H., Kim, H.S., Jang, I-T. (2020). Intervertebral Disc Diseases PART 2: A Review of the Current Diagnostic and Treatment Strategies for Intervertebral Disc Disease. Int J Mol Sci., 21 (6), 2135.

Zylbergold, R.S., Piper, M.C. (1981). Lumbar disc disease: comparative analysis of physical therapy treatments. Arch Phys Med Rehabil., 62 (4), 176-179.

Cite this article aS: Bolach, B., Walowska, J., Chabraszewska, P., Ryterska, K., Bolach, E. (2021). Possibilities of Using Outpatient Physiotherapy in the Process of Rehabilitation of People with Degenerative Disc Disease in the Lumbosacral Spine. Central European Journal of Sport Sciences and Medicine, 4 (36), 27-36. DOI: 10.18276/cej.2021.4-03. 